This document is the accepted manuscript version of the following article:

Hammoud, H., Valdivieso, F., \& Vaucher, S. (2018). Multiphysics modelling approach to microwave heating of cerium oxide particles in diverse packing situations. IET

Science, Measurement \& Technology. http://doi.org/10.1049/iet-smt.2017.0577

\title{
Multiphysics modelling approach to microwave heating of cerium oxide particles in diverse packing situations
}

\author{
Hussein Hammoud ${ }^{\mathrm{a}, \mathrm{b}}$, François Valdivieso ${ }^{\mathrm{a}}$,Sébastien Vaucher ${ }^{\mathrm{b}}$ \\ ${ }^{a}$ Laboratoire Georges Friedel CNRS UMR 5307, St-Etienne, France \\ ${ }^{\mathrm{b}}$ Swiss Federal Laboratories for Materials Science and Technology (EMPA), Thun, Switzerland
}

\begin{abstract}
In this work a single mode resonant microwave cavity $(2.45 \mathrm{GHz})$ is loaded with spherical particles. A weakly coupled Electromagnetic-Thermal (EM-thermal) solver is used iteratively to determine how the microscopic geometry (local curvatures between the particles, grain size and neck size) modifies the electromagnetic field, and in turn the thermal field in the particles and also to quantify the microwave effect for an experimental process. The modelling is performed with the conformal Finite Element (FE) solver COMSOL Multiphysics.

Moreover, this paper will show that the electric field norm increases for 1 spherical particle of ceria with different sizes and consequently the temperature increases. In addition, the electric field and heating behavior has been studied in the case of three particles with different neck sizes. Finally, the effect of the configuration versus the $\mathrm{E}$ field direction has been studied for three particles and has a significant result.
\end{abstract}

Keywords: EM-Thermal simulation, Cerium oxide, Microwave Processing, Effect of Neck Size, Packing configuration.

\section{Introduction}

The microwave technology is one of the candidates to improve the sintering of materials produced by powder technology. It enables a rapid, selective and volumetric heating of the green bodies that can be beneficial to the mechanical and physical properties of the final sintered parts [1,2]. Despite numerous efforts it remains unclear today how the local electromagnetic and thermal field gradients can influence the mass transfer mechanisms involved during sintering such as grain boundary diffusion and surface diffusion. Hao et al [3] suggest in their work about the microwave heating effect a model that includes the reduction in the formation energy of vacancy at the neck surface. Rybakov et al $[4,5]$ suggest a model to explain the nonthermal effect by a ponderomotive force which combines the diffusion mechanism and the electromigration fluxes of vacancies. On the other hand, Johnson shows in his work [6] that for grains diameters below $3 \mu \mathrm{m}$, the grain boundary diffusion predominates over volume diffusion for all sintering stages. In addition, Olevsky et al [7] conclude in their work that a considerable free surface electromigration appears during microwave sintering in a polarized electromagnetic field.

Though microwave technology can be used for the selective and rapid heating of many different classes of materials, sintering of ceramics is one of the largest potential for high temperature microwave applications [8].

Cerium dioxide (Ceria or $\mathrm{CeO}_{2}$ ) ceramic material find important uses, such as UV 
filters, electrolytes in SOFC (solid oxide fuel cell) technology and as non-radioactive uranium dioxide simulant in nuclear research, $[9,10]$.

Today, the availability of user-friendly softwares makes it attractive to address multiphysics problems such as microwave assisted sintering, in which the electromagnetic interactions are coupled to heat and mass transfer [11].

Therefore, in our work a coupled Electromagnetic-Thermal (EM-thermal) solver is used iteratively to determine how the microscopic geometry (local curvatures between the particles, grain size and neck size) modifies the electromagnetic field, and in turn the thermal field in the particles and also to quantify the microwave effect for a realistic process.

In this paper we use COMSOL Multiphysics to first explore numerically the effect of the size of a single spherical particle on the electric field magnitude and its heating behavior. In a second step we extend the model to a three particles system and explore additionally the influence of the contact point geometry and the effect of the configuration relative to the E-field axis. The dielectric and the thermal properties of cerium oxide (ceria) particles up to $1000^{\circ} \mathrm{C}$ have been determined in our previous study [12].

\section{Model and material parameters}

The scenario consist of a single mode resonating cavity based on a $260 \mathrm{~mm}$ segment of a rectangular waveguide WR340 ( $86 \mathrm{~mm} \times 43 \mathrm{~mm})$ delimited by a short circuit on one side and a symmetrical inductive rectangular iris $(65 \mathrm{~mm} \times 43 \mathrm{~mm})$ on the other side. The resonance of interest is a $\mathrm{TE}_{103}$ mode close to $2.45 \mathrm{GHz}$. The different physics (called modules in the software) such as electromagnetic module which is solved in frequency domain and the heat transfer in time domain are coupled together.

The figure 1 presents the geometry and the different elements of the cavity.

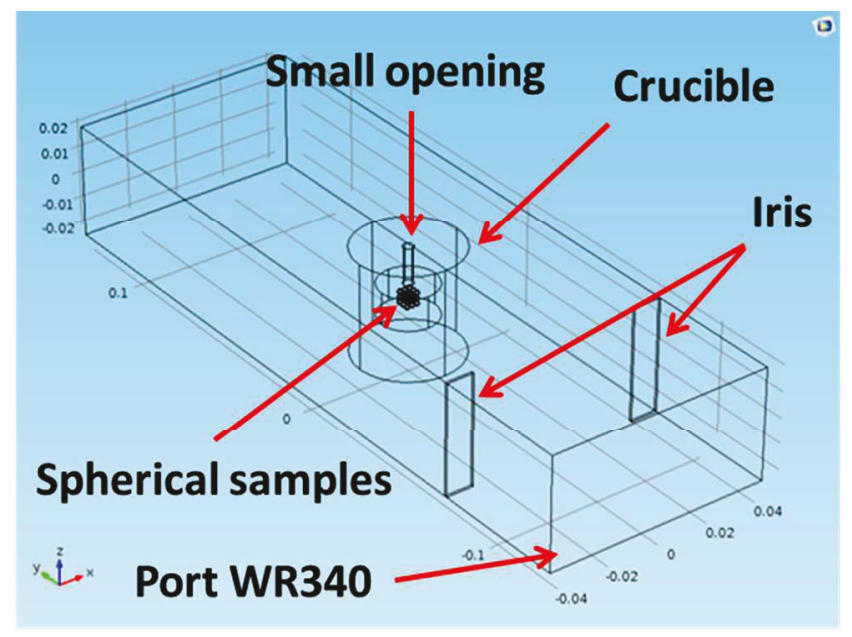

Figure 1: Geometry of the cavity and its elements (distance in meters).

The cavity is loaded with spherical ceria samples ( $\varnothing \quad 0.1-3 \mathrm{~mm}$ ) surrounded by a crucible, centered in one electric field maxima (see figure 2 ). The cavity walls are set at perfect metallic conductor. The microwave input power is fixed at $1000 \mathrm{~W}$ and the heating time at $2000 \mathrm{~s}$. The simulation process can be described as an iterative process where the multi-physics problem follows the different steps mentioned below:

First, an electromagnetic stationary calculation was made everywhere in the cavity and within the sample taking into account the dielectric properties (Equation 1) of the sample to provide the energy absorbed. The boundary conditions applied to the cavity walls supposed that they are 
perfectly conductors (Equation 2). Then, the energy absorbed by the sample acts as a local heat source (Equation 3). The heat transfer in the solid is also included in this study (Equation 4). The losses coming from the radiation of the sample surface are given by the Equation 5. Finally, the heat flow (Equation 6) assumed for the air was also included. The heat transfer and the heat losses were calculated in a time domain whereas the electromagnetic study was in frequency domain.

In this study, we were interesting to quantify the electric field in the whole cavity and the dissipation energy due to electric field contribution in the sample. The relative permeability of the different objects (sample, crucible ...) was fixed at 1; therefore the calculated losses are resulting

$$
\rho c_{p} \frac{\partial \boldsymbol{T}}{\partial \boldsymbol{t}}+\rho \boldsymbol{c}_{p} \boldsymbol{u} \cdot \nabla \boldsymbol{T}=\nabla .(\boldsymbol{k} \nabla \boldsymbol{T})+\boldsymbol{Q}_{s}
$$

(Eq.4)

Equation 4 (a) represents the general heat transfer in the solid (sample), and in our case, because sample is static the resulting equation becomes Eq.4 (b)

$$
\boldsymbol{\rho}_{f} \boldsymbol{c}_{p f} \frac{\partial \boldsymbol{T}}{\partial \boldsymbol{t}}+\boldsymbol{\rho}_{f} \boldsymbol{c}_{p f} \boldsymbol{u}_{f} . \nabla \boldsymbol{T}=\nabla .\left(\boldsymbol{k}_{f} \nabla \boldsymbol{T}\right)+\boldsymbol{Q}_{f}
$$

(a)

Equation 6 (a) represents the heat transfers in the air (the fluid) by conduction and convection. In our case convection is not taken into account and because air does not heat by microwave therefore the heat propagation equation becomes Equation.6 (b). from dielectric losses and the losses by conduction.

Now, we present the different equations used which relate the different physics involved:

$$
\nabla\left(\mu_{r}^{-1} \nabla \boldsymbol{E}\right)-\boldsymbol{k}_{0}^{2}\left(\boldsymbol{\varepsilon}_{r}-\frac{\boldsymbol{j} \boldsymbol{\sigma}}{\boldsymbol{\omega} \boldsymbol{\varepsilon}_{0}}\right) \boldsymbol{E}=0
$$

(Eq.1)

Equation 1 represents the wave propagation equation in the whole cavity.

$$
\vec{n} \wedge \vec{E}=\overrightarrow{0}
$$

(Eq.2)

Equation 2 represents the boundaries conditions at the cavity walls where the electric field is parallel, due to perfect conductive of constitutive material.

$$
P=\left(\sigma+2 \pi f \varepsilon_{0} \varepsilon_{e f f}^{\prime \prime}\right)|E|^{2}
$$

(Eq.3)

The electromagnetic dissipation in the materials is defined by equation 3

$$
\rho \boldsymbol{c}_{p} \frac{\partial \boldsymbol{T}}{\partial \boldsymbol{t}}=\nabla \cdot(\boldsymbol{k} \nabla \boldsymbol{T})+\boldsymbol{P}
$$

$$
-\boldsymbol{n} .(-\boldsymbol{k} \nabla \boldsymbol{T})=\boldsymbol{\varepsilon} \boldsymbol{\sigma}\left(\boldsymbol{T}_{a m b}^{4}-\boldsymbol{T}^{4}\right)(\text { Eq.5) }
$$

Equation 5 represents the radiative losses in the materials (ceria sample and alumina crucible).

$$
\boldsymbol{\rho}_{f} \boldsymbol{c}_{p f} \frac{\partial \boldsymbol{T}}{\partial \boldsymbol{t}}=\nabla \cdot\left(\boldsymbol{k}_{f} \nabla \boldsymbol{T}\right)
$$

(Eq.6)

with $|E|$ the electric field norm, $f$ the frequency, $\sigma$ is the electrical conductivity of ceria, $\varepsilon_{r}$ the relative dielectric permittivity of ceria, $\varepsilon_{e f f}^{\prime \prime}$ the imaginary part of the dielectric constant of ceria, $k, k_{f}$ the thermal conductivity of ceria and the air respectively, $\boldsymbol{c}_{p}, \boldsymbol{c}_{p f}$ the specific heat 
capacity of ceria and the air respectively, $\boldsymbol{u}$, $\boldsymbol{u}_{\boldsymbol{f}}$ the velocity field of ceria and the air, $\boldsymbol{\varepsilon}$ the emissivity of ceria, $\boldsymbol{Q}_{\boldsymbol{f}}, \boldsymbol{Q}_{s}$ the heat source for the air and the ceria respectively. $\boldsymbol{Q}_{\boldsymbol{f}}$ is equal 0 (case of air) and $\boldsymbol{Q}_{s}$ to $P$ (case of ceria).

The cavity walls have been imposed to be perfect electric conducting metal. The crucible material is based on porous alumina material. Its dielectric properties were very low in order to get as highest electric field magnitude as possible in the sample region. The relative permittivity and the thermal parameters of the ceria sample as function of temperature are given in the table 1:

\begin{tabular}{|cc|}
\hline Relative permittivity & Specific heat capacity (J/Kg.K) \\
$7.9519+0.0087 * T-j *\left(1.435410^{-14} * T^{5}-\right.$ & \\
$4.438810^{-11} * T^{4}+5.285610^{-8} * T^{3}-2.999110^{-5} * T^{2}$ & $5.42110^{-7} * T^{3}-1.438910^{-3} T^{2}$ \\
$\left.+8.078010^{-3} * T-0.8058\right)$ & $+1.2612 * T+62.208$ \\
Emissivity & Thermal conductivity (W/m.K) \\
$1.46310^{-7} * T^{2}-0.000310^{-3} * T+0.221$ & $-1.157 * \log (T)+8.7001$ \\
\hline
\end{tabular}

Table 1: Thermal and dielectric parameters of cerium oxide.

\section{Results and discussions}

\section{Mesh optimization:}

The selection of mesh element is important in such finite element solver because these elements are responsible for the provided information delivered by the software. Hence, the mesh element must be less than $\lambda_{\mathrm{g}} / 5$ in which $\lambda_{\mathrm{g}}$ is the guide wavelength and it is equal to $17.3 \mathrm{~cm}$. The reason is due to the discretization of the wave in order to ensure a better accuracy in the calculation. Otherwise, the results will depend strongly on the mesh.

The mesh was tested using many calculations in order to fix and optimize the mesh size in the sample region. It is important to know that the y axis is the axis through the length of the cavity, the $\mathrm{z}$ axis is the one through its height and the $\mathrm{x}$ axis is the one through its width.
Actually, the mesh elements were divided into three sizes depending on the concerned objects:

1- The maximum element size concerning the cavity was fixed at $\mathrm{Lz} / 6$ (7.2 $\mathrm{mm})$; with $\mathrm{Lz}$ is the height of the cavity.

2- The maximum element size concerning the spherical sample was fixed at $r / 10$ in volume; with $r$ is the sample radius.

3- Moreover, a different element size was applied to the sample surface (or boundary) in order to get more accuracy at the curvature region. Its value was $r / 15$.

In addition, four calculations of a ceria sphere with $100 \mu \mathrm{m}$ of radius are presented in figure 2 in order to quantify the effect of the mesh size on the electric field norm along y axis. 


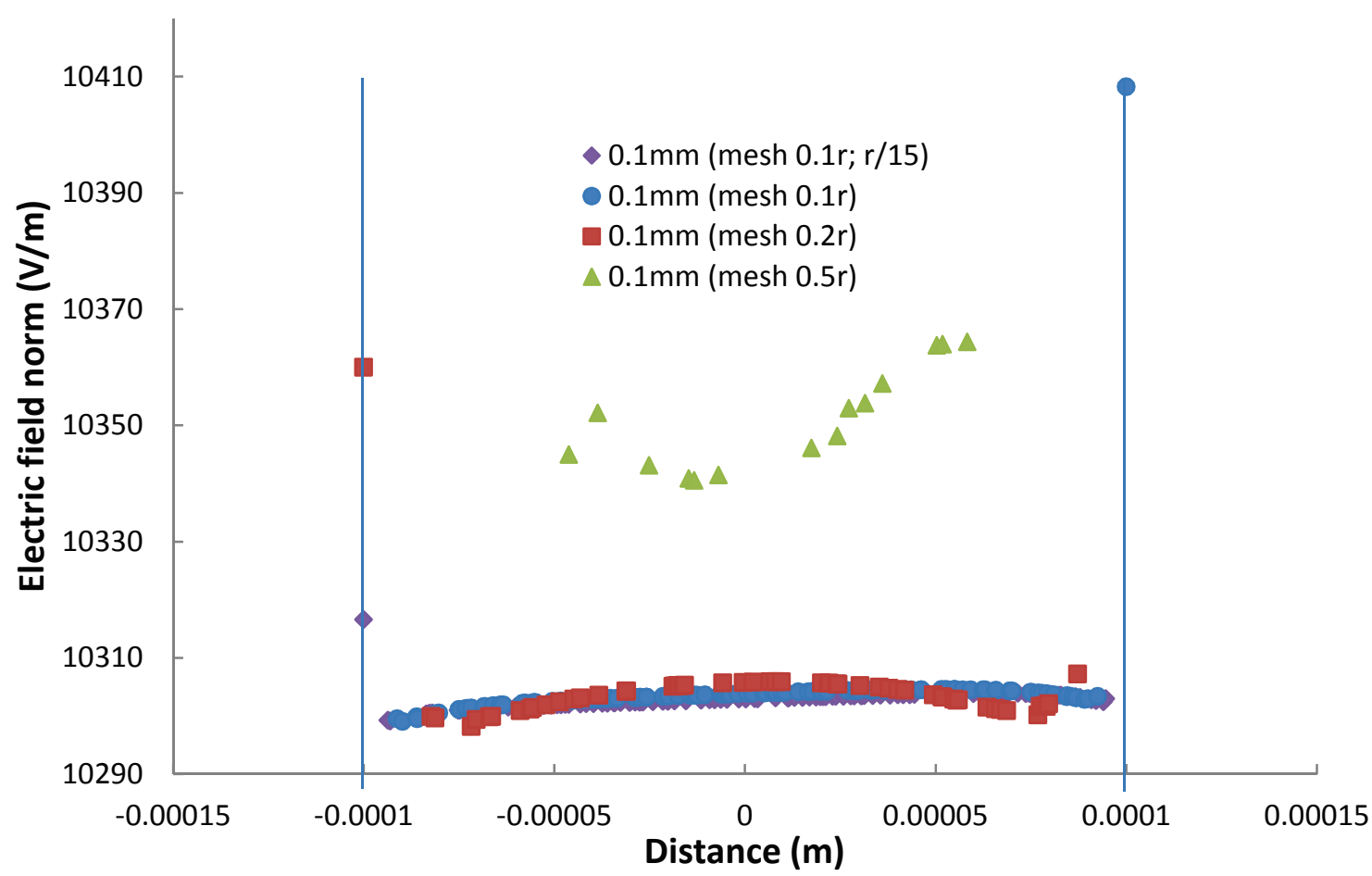

Figure 2: The variation of electric field magnitude through a spherical sample along y axis.

The element size of the mesh inside the sample has three maximum values $0.5 \mathrm{r}, 0.2 \mathrm{r}$ and $0.1 \mathrm{r}$ and in the fourth calculation the surface meshing was imposed to be $\mathrm{r} / 15$ with $0.1 \mathrm{r}$ inside the sphere. It is clear from the figure 2 that the accuracy is similar inside the centre of the sample for $0.1 \mathrm{r}$ and $0.2 \mathrm{r}$ (in blue and red) whereas the curve concerning the $0.5 \mathrm{r}$ (green triangles) is different. Moreover, the one with $0.1 \mathrm{r}$ and the imposed mesh at the surface (in purple) provides more precision at the interface as it appears lower on the left vertical line. We have chosen therefore the fourth mesh protocol which has these elements sizes:

1- $0.1 \mathrm{r}$ as maximum for the volume of the sample

2- $r / 15$ as maximum for its surfaces
3- $\mathrm{Lz} / 6$ as maximum for the cavity which is about $7.2 \mathrm{~mm}$.

\section{Effect of particle size:}

The objective of this calculation is to quantify the effect of particle size on the electric field magnitude and therefore define the optimal size in order to limit the calculation time. In this section, we present the results concerning the effect of particle size for a configuration of cavity with spherical ceria particle which is placed in the centre (figure 3 ). 
This article has been accepted for publication in a future issue of this journal, but has not been fully edited.

Content may change prior to final publication in an issue of the journal. To cite the paper please use the doi provided on the Digital Library page.

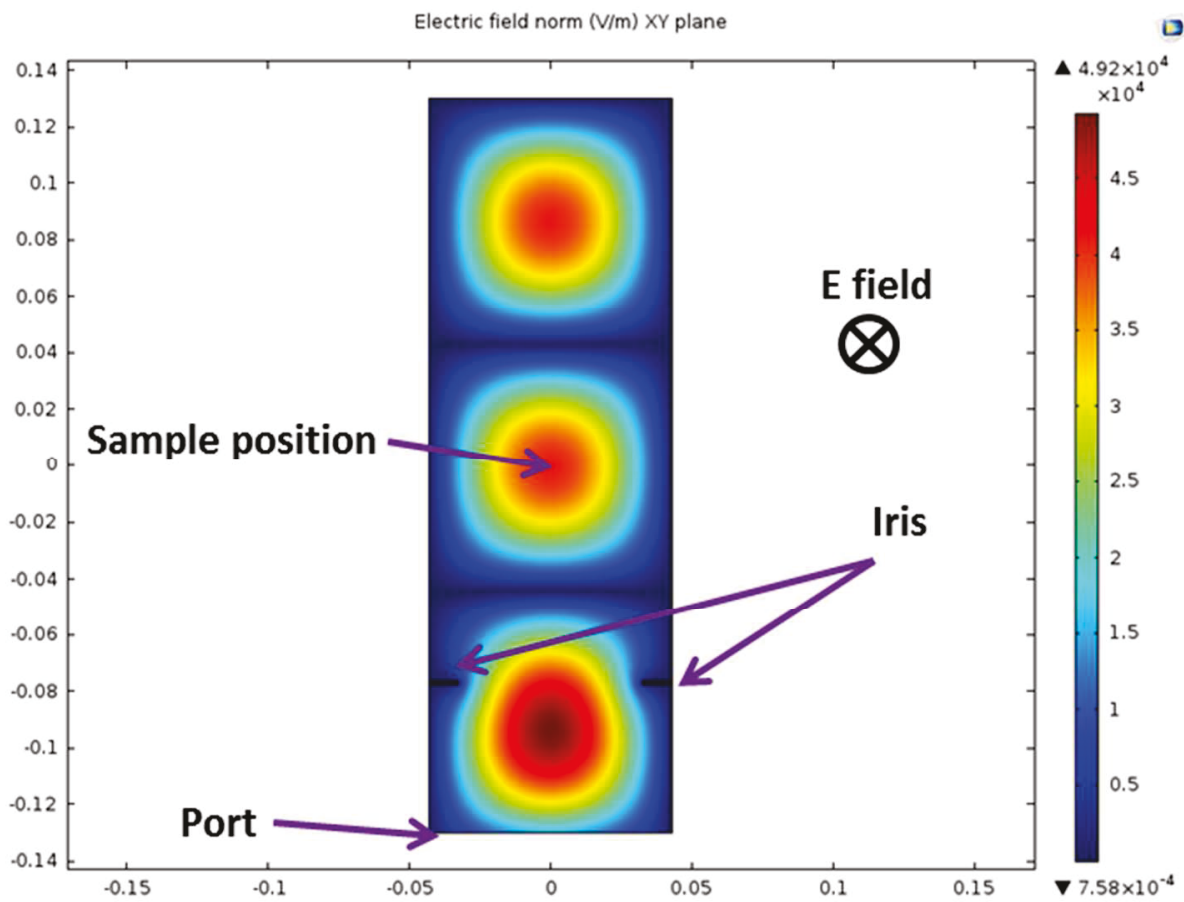

Figure 3: Maxima of the electric field magnitude in xy plane and the sample position.

The spherical sample which has the thermal and dielectric parameters of ceria, is placed in the centre of a region of high electric field magnitude. The particles radius varies from 0.01 to $1 \mathrm{~mm}$ due to the limits of dimensions of the software. The variation of the electric field norm along the $(\mathrm{x}, 0,0)$ and $(0, \mathrm{y}, 0)$ axis are presented in figure 4 , both are perpendicular to the E-field axis and along $(0,0, z)$ axis is presented in figure 5 which is parallel to E-field.

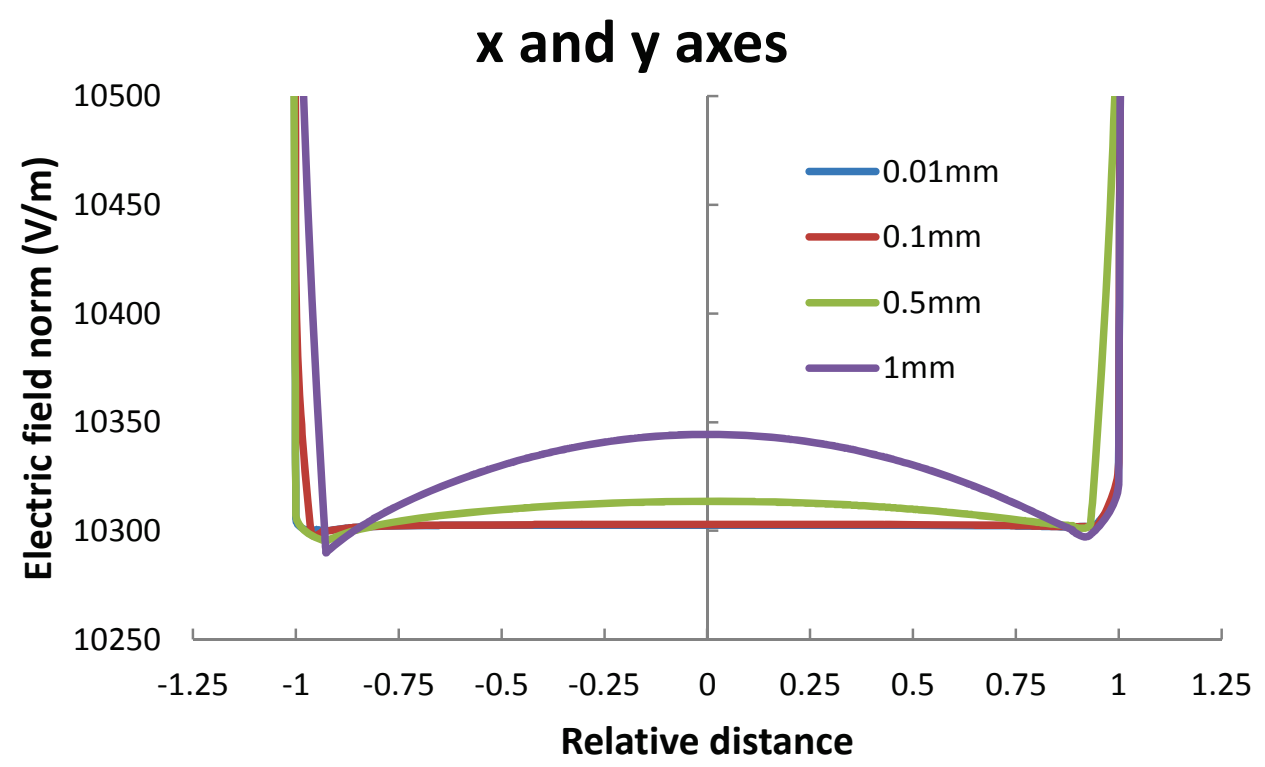

Figure4: The variation of electric field norm in the sample along $\mathrm{x}$ and $\mathrm{y}$ axis for four different sample radii $(0.01,0.1,0.5$ and $1 \mathrm{~mm})$. 


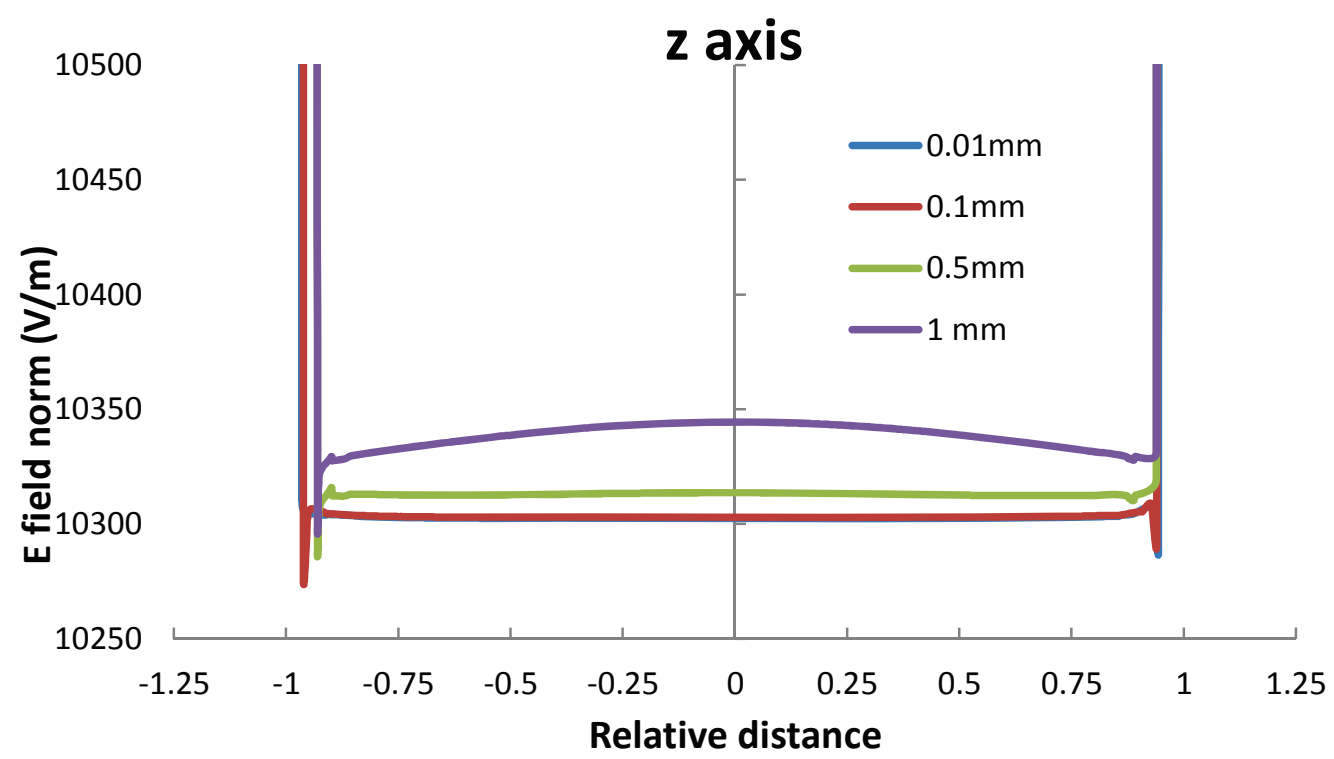

Figure 5: The variation of electric field norm in the sample along $\mathrm{z}$ axis for four different sample radii $(0.01,0.1,0.5$ and $1 \mathrm{~mm})$.

When the size of the particle increases the norm of E-field increases from $10300 \mathrm{~V} / \mathrm{m}$ as the maximum obtained at the centre of the smallest particle to 10350 for the largest one. Moreover, the E-field gradient through each particle is larger in $\mathrm{x}$ and $\mathrm{y}$ axis compared in $\mathrm{z}$ axis, but once the particle size decreases it remains constant; it will affect the heating. The spikes seen in this figure are just an artefact due to the discretization principle in the Finite Element method.

\section{Effect of neck size:}

Three spherical samples ( $0.1 \mathrm{~mm}$ of radius) are placed at the position on which the centre of the system has the same position as in case of one sphere. The ratio neck size over grain size $(\mathrm{X} / \mathrm{R})$ has three different values: 0 in case of no neck between the particles, 0.1 and finally 0.2 . The three particles are rearranged along y axis which is the axis along the length of the cavity (figure 6).

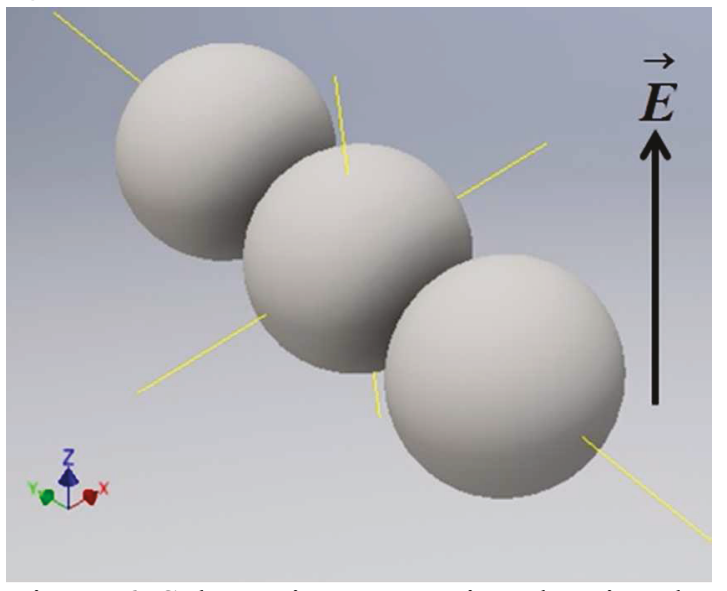

Figure 6: Schematic presentation showing the position of the system of three spheres $(0.1 \mathrm{~mm}$ of radius) and the axes in $\mathrm{Y}$ configuration.

The electric field magnitude in the particles varies from 7000 to $10000 \mathrm{~V} / \mathrm{m}$ and becomes very high near the surface 20000 $\mathrm{V} / \mathrm{m}$. For more precision, the figures $7-\mathrm{a}, 7-\mathrm{b}$ and 7-c present the variation of electric field magnitude along $\mathrm{x}, \mathrm{y}$ and $\mathrm{z}$ axis. 


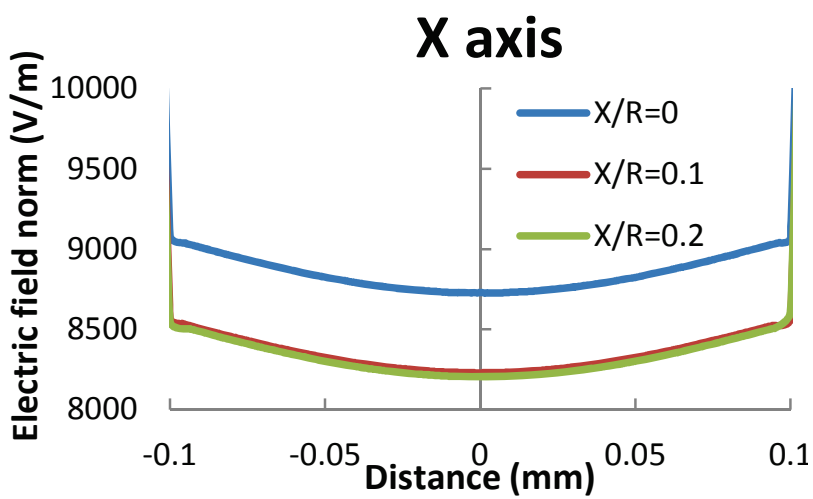

a)

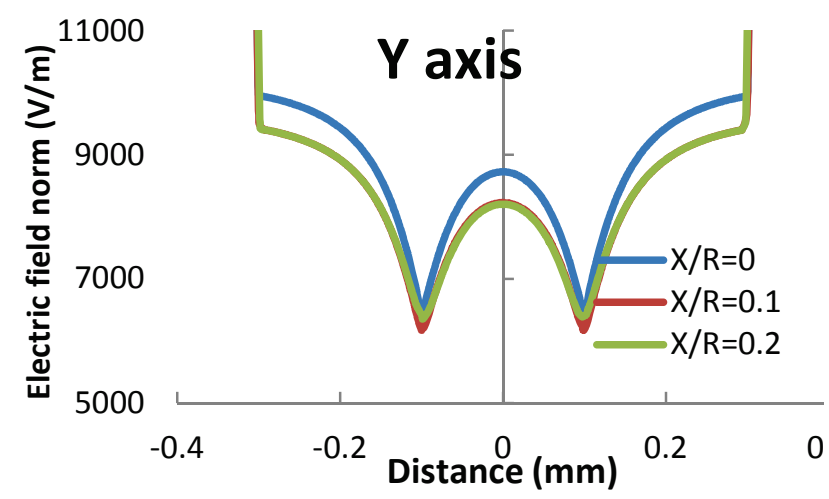

b)

The figure 7-a shows that the electric field along $\mathrm{x}$ axis decreases significantly from $8800 \mathrm{~V} / \mathrm{m}$ at the centre of the system (the central particle) to $8200 \mathrm{~V} / \mathrm{m}$ when the ratio neck size over grain size $(\mathrm{X} / \mathrm{R})$ increases from 0 to 0.2 . In terms of power, this variation corresponds to about $13 \%$ of the total dissipated power, using the equation 5.2. Moreover, a similar gradient of electric field norm through the sample for the three cases of $\mathrm{X} / \mathrm{R}$ ratio is presented and it is about $300 \mathrm{~V} / \mathrm{m}$.

The figure 7-b which presents the variation along $y$ axis through the three ceria particles. It shows the similar behaviour as in $\mathrm{x}$ axis at the centre of the central particle: the presence of neck in the packing decreases the electric field norm by about $600 \mathrm{~V} / \mathrm{m}$. Moreover, electric field difference is more important between the neck region and the

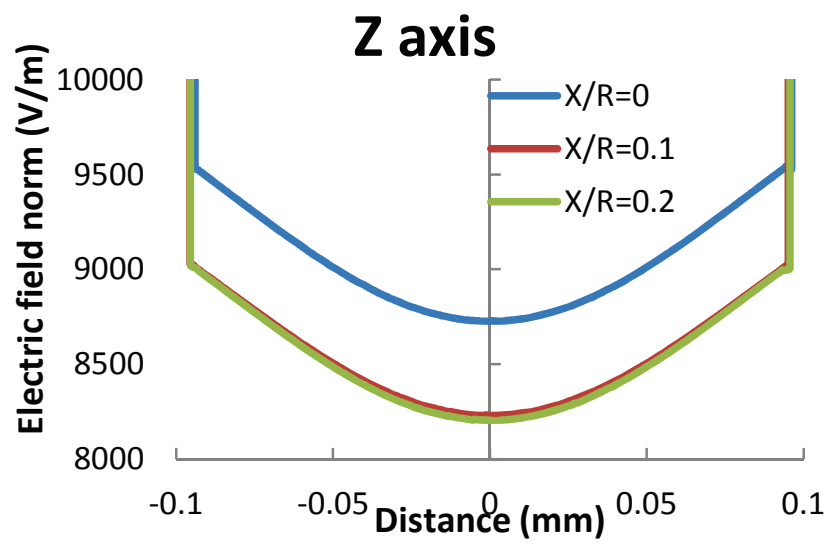

c)

Figure 7: The variation of electric field norm along $\mathrm{x}$ axis (a), y axis (b) and $\mathrm{z}$ axis (c) for three different neck sizes $(0,0.1$ and 0.2$)$. outer particles about $3500 \mathrm{~V} / \mathrm{m}$ than between the neck region and the centre of the central particle. The figure 7-c shows that the variation along $\mathrm{z}$ axis has the same behaviour as along $\mathrm{x}$ axis, except the E-field norm gradient which it is about $800.10^{4}$ $\mathrm{V} / \mathrm{m}^{2}$ between the centre and the surfaces of the central particle instead of $300.10^{4} \mathrm{~V} / \mathrm{m}^{2}$ along $\mathrm{x}$ axis.

\section{Effect of the packing orientation on the electric field:}

As previously, a model of three particles (a radius of $0.1 \mathrm{~mm}$ ) having no neck placed in a new configuration is presented in this section. The figure 8 shows a schematic presentation of the packing in $\mathrm{z}$ axis which is parallel to E-field direction (see figure 2). 
It is important to mention that the central particle of this packing has the same position as in the configuration along y axis (figure 5) which is perpendicular to the Efield direction.

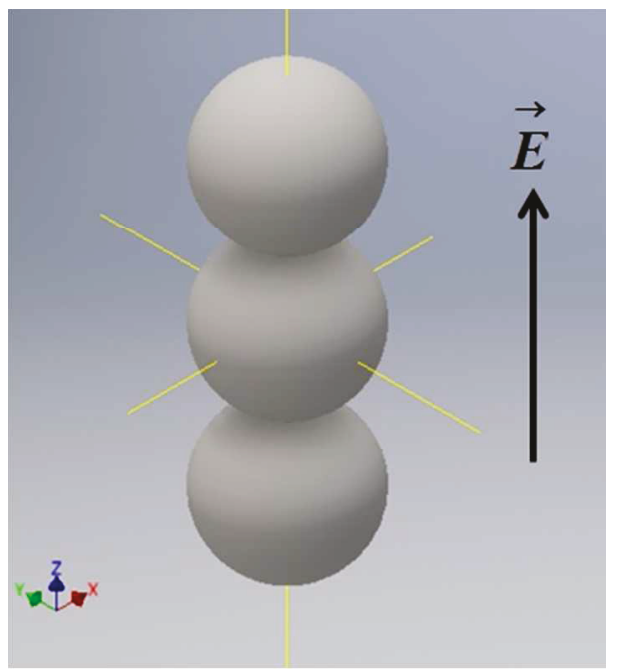

Figure 8: Schematic presentation showing the position of the system of three spheres $(0.1 \mathrm{~mm}$ of radius) and the axes in $\mathrm{Z}$ configuration.
The figures 9 and 10 present the variation of electric field magnitude and the temperature respectively for the two packing along their corresponding configuration axes (y axis for $\mathrm{Y}$ configuration and $\mathrm{Z}$ axis for $\mathrm{Z}$ configuration).

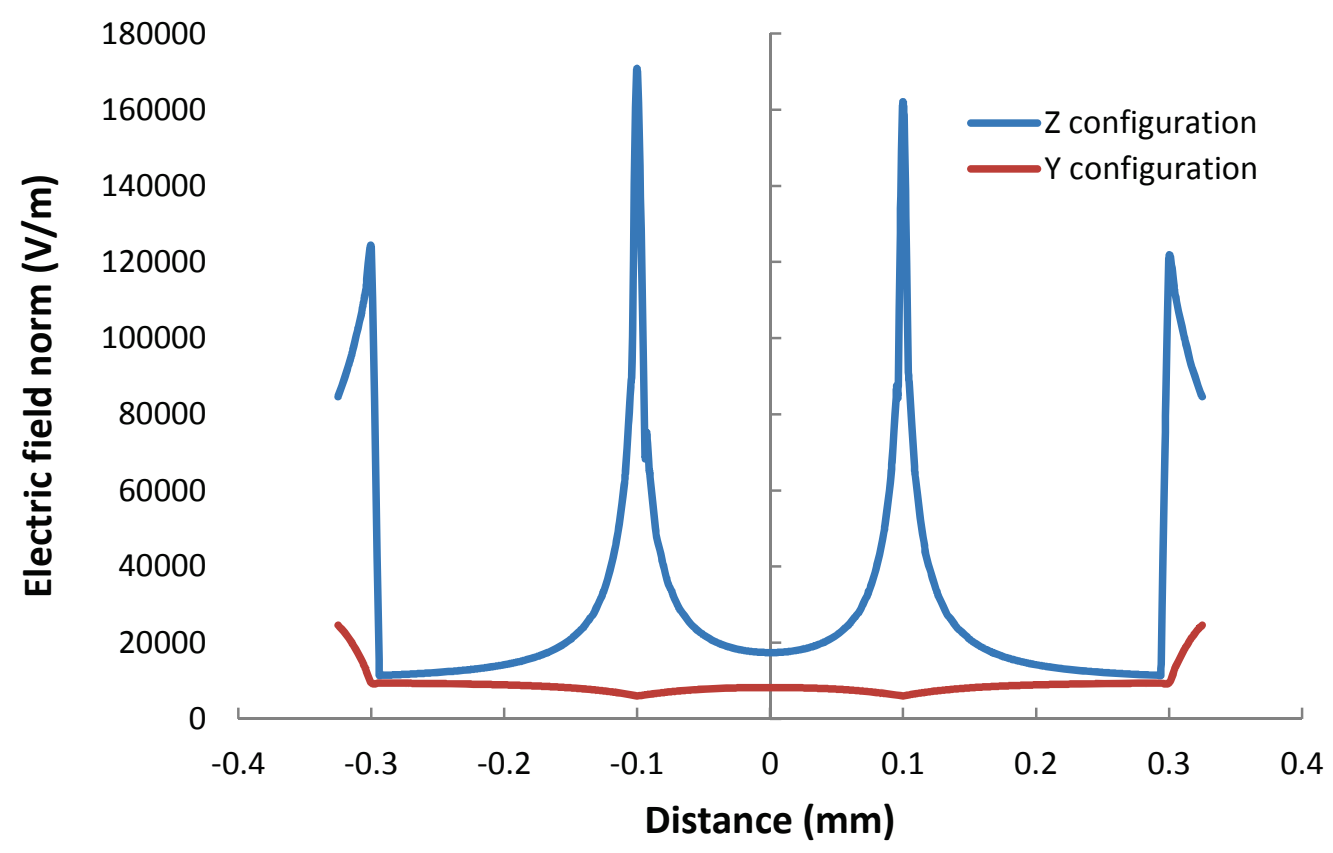

Figure 9: The variation of electric field norm for $\mathrm{Z}$ and $\mathrm{Y}$ configurations along packing.

The electric field (figure 9) is in general higher for $\mathrm{Z}$ configuration because this configuration is parallel to the $\mathrm{E}$ field direction. The value of the electric field in the centre of the central particle of the $\mathrm{Z}$ configuration is $17000 \mathrm{~V} / \mathrm{m}$ instead of 8000 
$\mathrm{V} / \mathrm{m}$ for $\mathrm{Y}$ configuration. Moreover, the electric field magnitude in $\mathrm{Z}$ configuration close to the neck region is much more important (about $170000 \mathrm{~V} / \mathrm{m}$ ) than the $\mathrm{Y}$ configuration which is equal to $6000 \mathrm{~V} / \mathrm{m}$ and the variation has a reverse behaviour when it passes from the centre of the system to the neck region. Moreover, comparing to the maximum E-field magnitude in the empty cavity (see figure 2), the electric field norm close to the neck region in $Z$ configuration is three times greater.

\section{Effect of the packing orientation on the heating}

The radiative losses are not yet taken into account in this configuration, only conductive losses are presented.

The variation of the temperature for the two packing orientations along their corresponding orientation axes is presented in figure 10.

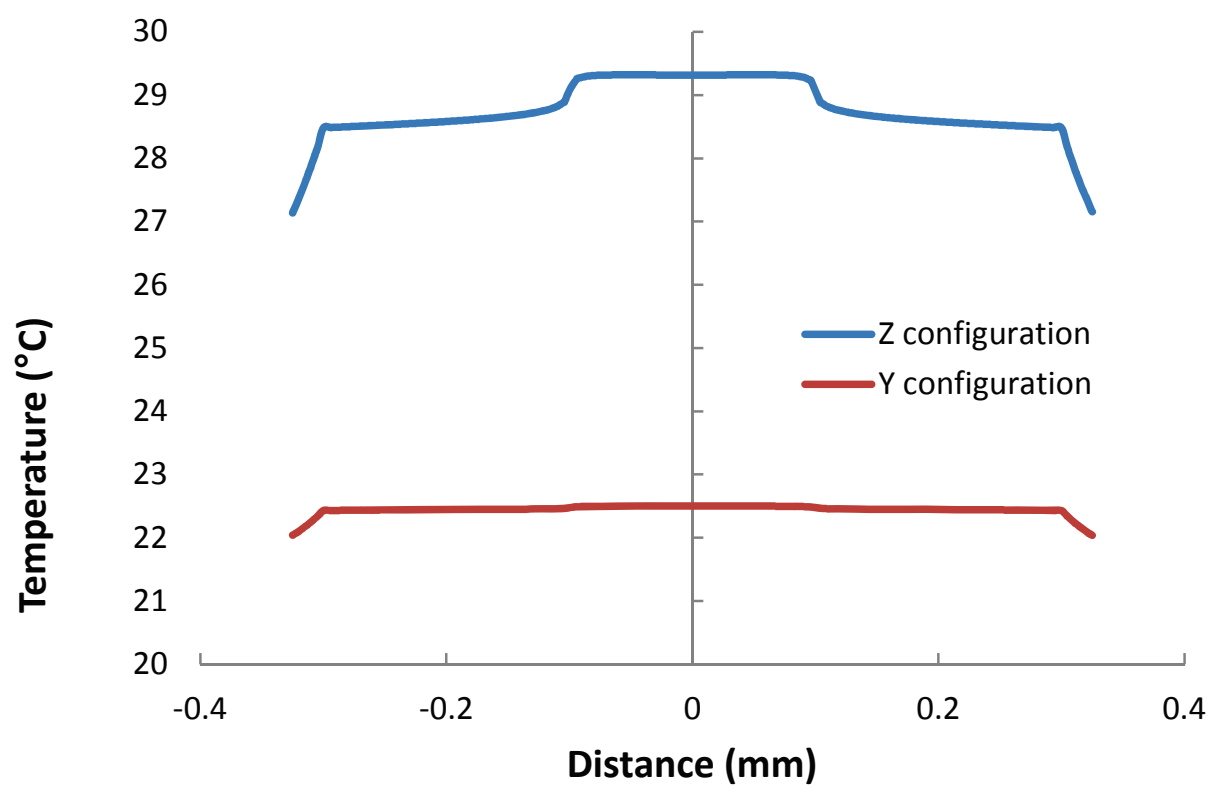

Figure 10: The variation of temperature for $\mathrm{Z}$ and $\mathrm{Y}$ configurations.

Concerning the temperature, $\mathrm{Z}$ configuration shows a higher value compared to $\mathrm{Y}$ configuration which agrees with the fact that the electric field is higher in $\mathrm{Z}$ configuration. But due to low size of the particles the temperature can attend not more than $30^{\circ} \mathrm{C}$ whereas for the same configurations of particles with a radius of $1 \mathrm{~mm}$ and in the presence of the crucible the temperature difference between the two configurations in the central particle is more than $400{ }^{\circ} \mathrm{C}$ (figure 11). 


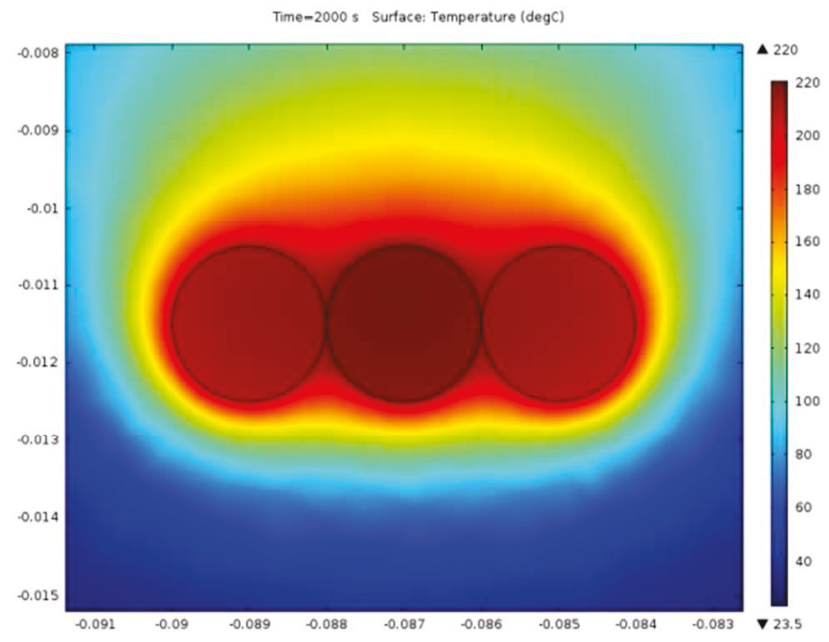

a)

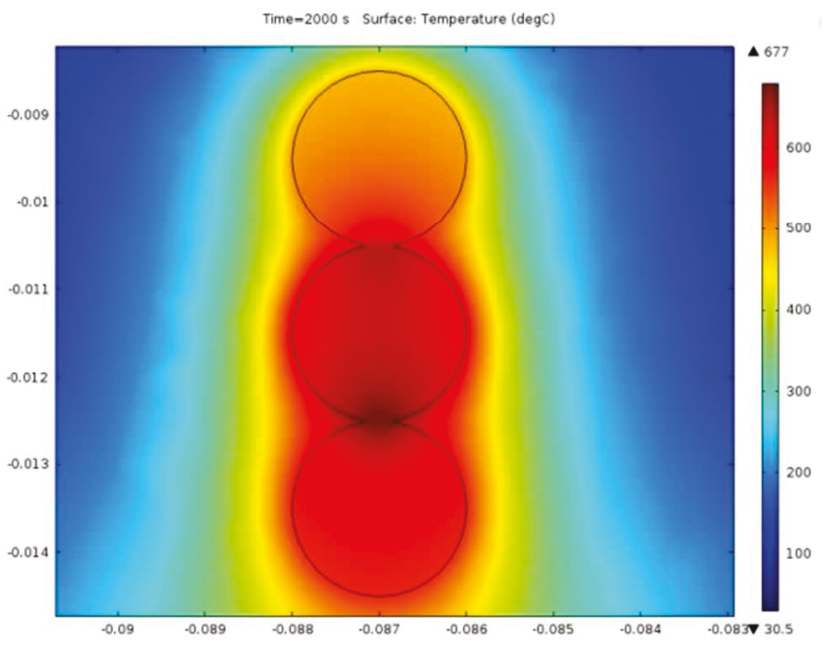

b)

Figure 11: the variation of temperature within a cross section (in yz) of the three particles: (a) in $Y$ configuration and (b) in $\mathrm{Z}$ configuration.

The centre of the central particle has $220^{\circ} \mathrm{C}$ in case of $\mathrm{Y}$ configuration and $629{ }^{\circ} \mathrm{C}$ in case of $\mathrm{Z}$ configuration. As this system doesn't take into account the radiative and the convective losses, this drop in temperature results from the material weight which is 1000 times lower for $0.2 \mathrm{~mm}$ of particle size compared to $2 \mathrm{~mm}$ of particle size.

According to the results obtained for the two packing orientations, we can deduce that the $\mathrm{Z}$ configuration leads to an intensification of the electric field and consequently a higher heating. The significant modification of the electric field leads to different temperatures depending on the orientation of the packing. Therefore, we could expect a differential sintering according to this inhomogeneous situation, like Whittaker [13] who supports the idea that the mass transport mechanism during the sintering of ceramic evolves preferentially along the electric field vector.

\section{Conclusion}

In this work, we present a model of single mode cavity with conditions of microwave heating process. The results show that for a single particle, the particle size has a significant effect on the electric field. In addition, for the three particles case, the presence of a neck modified the electric field magnitude which would have an influence on the densification mechanism. Finally, the results obtained by this Multiphysics simulation confirmed that the microwave heating is possible for ceria particles packing and it has a different interaction according to the orientation of interfaces with respect to the E-field direction.

\section{References}

[1] P. Yadoji, R. Peelamedu, D. Agrawal, R. Roy.: 'Microwave sintering of $\mathrm{Ni}-\mathrm{Zn}$ ferrites: comparaison with conventional sintering', Materials Science and Engineering B 98, 2003, 269-278. 
[2] D. Agrawal.: 'Microwave sintering of ceramics, composites, metals, and transparent materials', Journal of Materials Education, 1999, 19 4958.

[3] H. Hao et al.: 'Kinetics mechanism of microwave sintering in ceramic materials', Sci China Ser E-Tech Sci, 2009, vol. 52, 2727-2731.

[4] K. I. Rybakov and V. E. Semenov,: 'Possibility of plastic deformation of an ionic crystal due to the nonthermal influence of a highfrequency electric field', Physical Review B, 1994, vol. 49, 64-68,

[5] K. I. Rybakov and V. E. Semenov.: 'Mass transport in ionic crystals induced by the ponderomotive action of a high-frequency electric field', Physical Review B, 1995, vol. 52, 3030-3033.

[6] D.L. Johnson.: 'Comment on Temperature-Gradient-Driven Diffusion in Rapid-Rate Sintering, J. Am. Ceram. Soc., 1990, 73(8), 2576-2578.

[7] E. Olevsky et al.: 'Ponderomotive effects during contact formation in microwave sintering', Modelling Simul. Mater.Sci. Eng. 2013, 21.

[8] W. H. Sutton.: 'Microwave processing of ceramic materials', Am. Ceram. Soc, 1989, 68, 376-86.

[9] M. Jalilpour and M. Fathalilou.: 'Effect of aging time and calcination temperature on the cerium oxide nanoparticles synthesis via reverse co-precipitation method', International $\mathrm{J}$. of the Physical Sciences, 2012, vol. 7(6), 944-948.

[10] P.D Edmondson.: 'Fundamentals of the behavior of fission products in oxide nuclear fuels', 2014, University of Oxford.

[11] D. Bouvard et al.: 'Multiphysics Simulation of Microwave Sintering in a Monomode Cavity', 12th Seminar Computer Modeling in Microwave Engineering \& Applications, Grenoble, France, 2010.

[12] H. Hammoud, S. Vaucher and F. Valdivieso.: 'Dielectric and thermal properties of cerium dioxide up to $1000^{\circ} \mathrm{C}$ and the effect of the porosity for microwave processing studies', Thermochimica Acta, 2015, vol. 617, 83-89.

[13] A. G. Whittaker.: 'Diffusion in Microwave-Heated Ceramics', Chem. Mater., 2005, 17, 3426-3432. 